\title{
The discriminative stimulus effects of dopamine D2- and D3-preferring agonists in rats
}

\author{
Mikhail N. Koffarnus • Benjamin Greedy • \\ Stephen M. Husbands • Peter Grundt • \\ Amy Hauck Newman • James H. Woods
}

Received: 2 June 2008 / Accepted: 30 August 2008 / Published online: 21 September 2008

(C) Springer-Verlag 2008

\begin{abstract}
Rationale Previous research has found the stimulus effects of dopamine D2- and D3-preferring agonists difficult to distinguish in drug discrimination studies. Antagonism studies suggest that the stimulus effects of both types of agonists may be mediated primarily through D2 receptors. Objectives The current study was designed to further assess the receptors mediating the stimulus effects of these agonists and to attempt to train rats to discriminate directly between D2- and D3-preferring dopamine agonists.
\end{abstract}

M. N. Koffarnus $(\square) \cdot$ J. H. Woods

Department of Psychology, University of Michigan,

1301 MSRB III, 1150 W Medical Center Dr.,

Ann Arbor, MI 48109, USA

e-mail: mickyk@umich.edu

J. H. Woods

Department of Pharmacology, University of Michigan,

1301 MSRB III, 1150 W Medical Center Dr.,

Ann Arbor, MI 48109, USA

B. Greedy $\cdot$ S. M. Husbands

Department of Pharmacy and Pharmacology, University of Bath,

Claverton Down,

Bath BA2 7AY, UK

P. Grundt

Department of Chemistry and Biochemistry,

University of Minnesota Duluth,

1039 University Drive,

Duluth, MN 55812, USA

\section{A. H. Newman}

Medicinal Chemistry Section,

National Institute on Drug Abuse-Intramural Research Program,

National Institutes of Health,

5500 Nathan Shock Drive,

Baltimore, MD 21224, USA
Materials and methods Four groups of eight rats were trained to discriminate either $0.1 \mathrm{mg} / \mathrm{kg}$ of the D3preferring agonist pramipexole from saline, $1.0 \mathrm{mg} / \mathrm{kg}$ of the D2-preferring agonist sumanirole from saline, $0.1 \mathrm{mg} / \mathrm{kg}$ pramipexole from either saline or $1.0 \mathrm{mg} / \mathrm{kg}$ sumanirole, or $1.0 \mathrm{mg} / \mathrm{kg}$ sumanirole from either saline or $0.1 \mathrm{mg} / \mathrm{kg}$ pramipexole.

Results Three of eight rats in the $0.1 \mathrm{mg} / \mathrm{kg}$ pramipexole vs. $1.0 \mathrm{mg} / \mathrm{kg}$ sumanirole or saline failed to meet the training criteria, and the discrimination in this group was tenuous. The D2-preferring antagonist L-741,626 at $1.0 \mathrm{mg} / \mathrm{kg}$ was more effective at shifting to the right the pramipexole dose-response curve in pramipexole-trained rats, while $32 \mathrm{mg} / \mathrm{kg}$ of the selective D3 antagonist PG01037 had little effect. Quinpirole and 7-OH-DPAT fully or partially substituted for both pramipexole and sumanirole in each group tested, while cocaine did not substitute in any group.

Conclusions Antagonist data along with the pattern of training and substitution data suggested that D2 receptor activation is primarily responsible for the stimulus effects of both sumanirole and pramipexole with D3 receptor activation playing little or no role.

Keywords Drug discrimination - Either/or discrimination . D2 · D3 · Dopamine · Pramipexole · Sumanirole · Rat

Regardless of their selectivity for D2 or D3 receptors, a wide variety of D2-like agonists share discriminative stimulus effects (Katz and Alling 2000). These discriminative stimulus effects have been proposed to be dependent on both D3 activation and D2 activation. For example, a study correlating D3 receptor activation in vitro with in vivo potency to substitute for a discriminative stimulus elicited by the D3-preferring compound 7-OH-DPAT 
concluded that D3 receptors likely mediated the stimulus effects of this compound (Varty and Higgins 1997). However, studies employing more selective D2 and D3 antagonists have concluded the opposite, suggesting that the discriminative stimuli elicited by the D3-preferring agonists S32504 (Millan et al. 2007), PD 128,907 (Bristow et al. 1998; Millan et al. 2000), and 7-OH-DPAT (Millan et al. 2000; Christian et al. 2001) are mediated through D2 receptors. For example, Millan et al. (2007) have recently reported that the discriminative stimulus elicited by the D3preferring agonist S32504 is unaffected by pretreatments with the D3-preferring antagonist S33084, but is antagonized by the D2-preferring antagonist L-741,626 and the D2/D3/D4 antagonist haloperidol.

Elicitation of yawning behavior and hypothermia has been shown to correlate with in vivo potency at D3 and D2 receptors, respectively (Collins et al. 2007). This research has highlighted the discrepancies between the in vivo and in vitro selectivity of D3-preferring compounds. For example, the D3-preferring agonist pramipexole is approximately 170 -fold selective for D3 receptors compared to D2 and D4 in an in vitro binding assay (Newman-Tancredi et al. 2002), but was only 32-fold selective in vivo when comparing elicitation of yawning behavior and hypothermia (Collins et al. 2007).

The present set of experiments was designed to measure if and to what degree D2 and D3 receptors mediate the discriminative stimulus produced by $\mathrm{D} 2 / \mathrm{D} 3$ agonists by using selective antagonists and a procedural variation on the standard drug discrimination paradigm. One group of rats was trained to discriminate the D3-preferring agonist pramipexole (D3/D2 selectivity of 170-fold in vitro, NewmanTancredi et al. 2002; 32-fold in vivo, Collins et al. 2007) from saline. A second group of rats was trained to discriminate the D2-preferring agonist sumanirole (D2/D3 selectivity of 216-fold in vitro, McCall et al. 2005; approximately 19-fold in vivo, Collins et al. 2007) from saline. The training dose of pramipexole was $0.1 \mathrm{mg} / \mathrm{kg}$, the dose that elicits maximal levels of the D3-mediated behavior of yawning; and the training dose of sumanirole was $1.0 \mathrm{mg} / \mathrm{kg}$, a dose that has been shown to induce a robust D2-mediated hypothermia response (Collins et al. 2007). The D2-preferring antagonist L-741,626 (D2/D3 selectivity of 15-fold in vitro, Grundt et al. 2007a), the D3preferring antagonist PG01037 (D3/D2 selectivity of 133fold in vitro, Grundt et al. 2007b), and the nonselective D2/ D3/D4 antagonist haloperidol was used to determine the receptor subtypes mediating the pramipexole and sumanirole discriminative stimuli. Additional compounds tested include cocaine and the D3-preferring agonists quinpirole and 7-OHDPAT.

Two additional groups of rats were trained on an "either/ or" procedure. The first group was trained to discriminate sumanirole from either pramipexole or saline, and the second group was trained to discriminate pramipexole from either sumanirole or saline. This procedure was designed to train rats to distinguish the discriminative stimuli produced by individual receptor subtypes using compounds that have some affinity for both (Overton 1982). With the premise that a ligand like pramipexole, with affinity for both D3 and D2 receptors, produces a complex stimulus mediated through both receptors, the D2 component of this compound stimulus can be, in theory, "subtracted out" by associating the D2-preferring agonist sumanirole with the response option opposite pramipexole. This procedure has been successfully employed in the past to separate the discriminative stimulus effects mediated by $\mathrm{GABA}_{\mathrm{A}}$ and $\mathrm{GABA}_{B}$ receptor subtypes (Koek et al. 2005). Unlike procedures that associate each of three injections with distinct responses (e.g., Caul et al. 1996), the either/or procedure uses only two manipulanda and responses and can be carried out in relatively abundant two-response operant chambers. A caveat of this procedure, however, is that it is sometimes difficult to determine which drug stimulus is approximated by an injection when responses are made on the compound stimulus manipulandum.

\section{Materials and methods}

\section{Subjects}

Four groups of eight male Sprague Dawley rats served as subjects (Harlan, Indianapolis, IN, USA). Rats were approximately 10 weeks old at the start of the experiment. A food restriction protocol was in place to maintain the rats at approximately $325 \mathrm{~g}$ throughout the experiment. This weight was chosen as it is approximately $85 \%$ of the mean adult weight supplied by the manufacturer for this strain, and this weight was not changed once established. When not in session, rats were housed in accordance with institutional animal care and use guidelines in polycarbonate cages with fresh water continuously available. The lights in the housing colony were on from 7:00 A.M. to 7:00 P.M., and sessions were conducted between 1:00 P.M. and 6:00 P.M. These protocols were approved by the University of Michigan Committee on the Use and Care of Animals and conformed to the guidelines established by the NIH Guide for the Use of Laboratory Animals.

\section{Apparatus}

Sessions were conducted in rodent operant conditioning chambers with an area of $30.5 \mathrm{~cm} \times 24.1 \mathrm{~cm} \times 21.0 \mathrm{~cm}$ and stainless steel grid floors (ENV-008; Med-Associates, St. Albans, VT, USA). Both sides of the front panel of the 
chamber held a nose-poke response device $2.5 \mathrm{~cm}$ in diameter back-lit with a yellow LED light (ENV-114BM, Med-Associates). Between the nose-poke devices was an aperture containing a yellow LED light and a dipper capable of bringing $50 \mu \mathrm{l}$ of a fluid within reach of the rat (H14-05R; Coulbourn Instruments, Whitehall, PA, USA).

\section{Procedure}

Each daily session began with the illumination of both nose-poke holes. Subjects were trained to respond on a fixed ratio (FR) 1 schedule of reinforcement on the left or right nose-poke holes on alternating days, with the opposite hole remaining inactive. The FR was gradually increased to FR 15, and the response requirement reset to the FR value if the rat switched nose-poke holes before completing a ratio. During training sessions, an FR completion on the injection-appropriate hole resulted in 10-s access to $50 \mu \mathrm{l}$ Ensure (vanilla flavor, delivered undiluted), followed by a 15-s timeout. An FR completion in the opposite hole led to the 15-s timeout only. Test sessions were identical, except a completed FR in either hole led to 10-s access to Ensure. Sessions ended after 20 trials or $20 \mathrm{~min}$, whichever occurred first. If responding was such that all 20 trials resulted in the presentation and consumption of an Ensure delivery, up to $1 \mathrm{ml}$ of Ensure could be earned per session.

Injections were given $5 \mathrm{~min}$ prior the start of each session, and the rat was immediately placed in a darkened chamber. Antagonist pretreatments, where applicable, were given $30 \mathrm{~min}$ prior to the initiation of a session $(25 \mathrm{~min}$ prior to the agonist or saline injection and the rat being placed in the darkened chamber). Training conditions differed in each of four groups and are detailed in Table 1. Two groups were trained to discriminate a single drug from saline: $0.1 \mathrm{mg} / \mathrm{kg}$ pramipexole from saline or $1.0 \mathrm{mg} / \mathrm{kg}$ sumanirole from saline. The other two groups were trained on an either/or procedure, in which one nose-poke hole was associated with two different injections. One of these groups was trained to discriminate $0.1 \mathrm{mg} / \mathrm{kg}$ pramipexole from a rotation of $1.0 \mathrm{mg} / \mathrm{kg}$ sumanirole and saline, and the other group was trained discriminate $1.0 \mathrm{mg} / \mathrm{kg}$ sumanirole from a rotation of $0.1 \mathrm{mg} / \mathrm{kg}$ pramipexole and saline. To minimize the probability of side biases developing, the sequence of training days in all four groups was arranged such that the injection-associated nose-poke hole switched from left to right or vice versa each training session. In the either/or groups, this resulted in fewer training sessions with two of the injections, but an equal number of sessions with the left and right nose-poke responses being reinforced.

Criteria for injection-appropriate responding during a training session were first, $\geq 85 \%$ of the responses during the first trial and entire session emitted on the injection-associated nose-poke hole, and second, $\geq 60$ total responses during the session. The first test session for which the data was used in statistical analyses and graphical presentation was conducted after five consecutive successful training sessions and a successful test session using each of the two or three training injections, each of which followed two successful training sessions. Test sessions, after the first, were conducted after two consecutive successful training sessions.

\section{Drugs}

Pramipexole was provided by Drs. Jianyong Chen and Shaomeng Wang (University of Michigan, Ann Arbor, MI, USA), sumanirole by Benjamin Greedy and Dr. Stephen Husbands (University of Bath, Bath, UK), and PG01037 by Drs. Amy H. Newman (Medicinal Chemistry SectionNIDA, Baltimore, MD, USA) and Peter Grundt (University of Minnesota Duluth, Duluth, MN, USA). Quinpirole, 7-OHDPAT, and haloperidol were obtained from Sigma-Aldrich (St. Louis, MO, USA), L-741,626 was obtained from Tocris (Ellisville, MO, USA), and cocaine was obtained from the National Institute on Drug Abuse (Bethesda, MD, USA). All drugs were dissolved in sterile saline except L-741,626, which was dissolved in 5\% ethanol, and PG01037, which was dissolved in 20\% $\beta$-cyclodextrin. All drugs were administered subcutaneously in a volume of $1.0 \mathrm{ml} / \mathrm{kg}$.

\section{Data analysis}

Percent response data from any test session were included if $\geq 30$ total responses were recorded, and these data were used for any dose if three or more subjects met this 30-response

Table 1 Training conditions and mean sessions required to meet training criteria for each group

\begin{tabular}{llllc}
\hline Left & Right & Side sequence & Drug sequence & Sessions to criteria $( \pm$ SEM) \\
\hline 0.1 pram & sal & L-R-L-R $\ldots$ & pram-sal-pram-sal... & $110( \pm 16.6)$ \\
1.0 sum & sal & L-R-L-R $\ldots$ & sum-sal-sum-sal... & $66( \pm 7.1)$ \\
0.1 pram & 1.0 sum or sal & L-R-L-R ... & pram-sum-pram-sal... & $139( \pm 13.0)^{\mathrm{a}}$ \\
1.0 sum & 0.1 pram or sal & L-R-L-R ... & sum-pram-sum-sal... & $129( \pm 15.6)$ \\
\hline
\end{tabular}

pram Pramipexole, sum sumanirole, $L$ left, $R$ right

${ }^{a}$ Only five of eight rats met training criteria. The other three rats experienced 310, 143, and 150 sessions, respectively, before being dropped from the experiment. 
criterion. A drug was considered to fully substitute for the training drug if $\geq 75 \%$ of responses were recorded on the drug-appropriate nose-poke hole, while $\leq 25 \%$ responding was considered to be no substitution. All data were included in response rate analyses and graphical presentations. Linear regression analyses were conducted with GraphPad Prism 5 (La Jolla, CA, USA) to determine if dose was significantly correlated with percent drug-appropriate responding or response rate and also to determine the ED50 values for those measures. Comparisons of regression lines were also conducted to determine if antagonist pretreatments significantly shifted agonist substitution curves. This was done using Prism 5, which employs a method mathematically equivalent to an Analysis of Covariance (for a detailed description of this method, see Motulsky and Christopoulos 2003; Zar 1999, chap 18). Sessions to meet training criteria, as described above, were compared between groups with a one-way analysis of variance in Systat SigmaStat 3.5 (San Jose, CA, USA).

\section{Results}

All rats met training criteria, except three rats in the group trained to discriminate $0.1 \mathrm{mg} / \mathrm{kg}$ pramipexole from either $1.0 \mathrm{mg} / \mathrm{kg}$ sumanirole or saline. The mean sessions to meet criteria are shown in Table 1. These data represent the total number of sessions, including initial sessions in which the rats were trained to respond to the nose-poke devices on an FR 15 schedule, during which training injections were also given. There was a significant effect of training group on sessions to meet criteria $[F(3,25)=5.35, p=0.006]$. The group trained to discriminate $1.0 \mathrm{mg} / \mathrm{kg}$ sumanirole from saline required approximately half the sessions to meet training criteria compared with the other three groups, which was significantly less than the pramipexole vs. sumanirole or saline group and the sumanirole vs. pramipexole or saline group (Bonferroni-corrected post hoc tests, both $p<0.015$ ). No other training group differed significantly from any other with regard to sessions required to meet training criteria.

In the group trained to discriminate $0.1 \mathrm{mg} / \mathrm{kg}$ pramipexole from saline, only pramipexole and sumanirole fully substituted for the pramipexole stimulus at some dose (Fig. 1, top panel). Quinpirole and 7-OH-DPAT produced a partial substitution, and cocaine did not substitute for pramipexole at any dose tested. The slopes of the pramipexole $[F(1,30)=28.9$, $p<0.001]$ and sumanirole $[F(1,26)=10.0, p=0.004]$ substitution curves were significantly nonzero, indicating a significant effect of dose on responding to the pramipexoleassociated nose-poke hole (see Table 2 for ED50 values). Each of the D2-like agonists dose dependently reduced rates of responding, while cocaine did not alter response rates at
0.1 Pramipexole | Saline
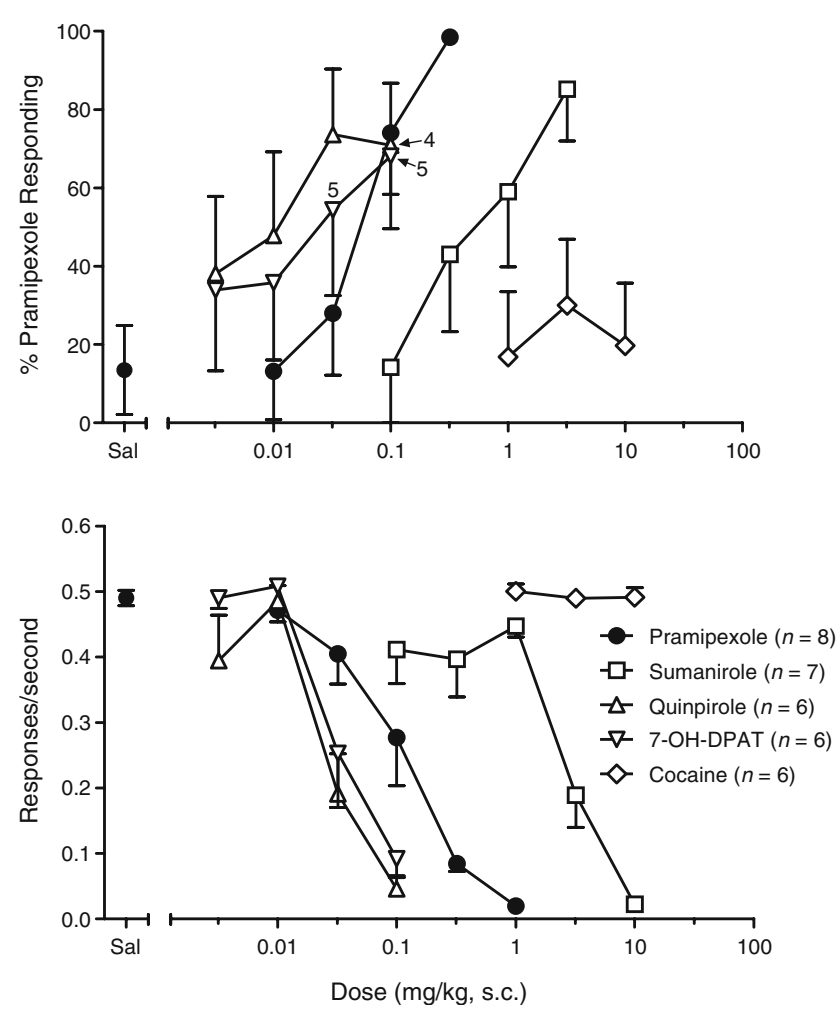

Fig. 1 Substitution and rates of responding engendered by dopamine D2-like agonists and cocaine in rats trained to discriminate $0.1 \mathrm{mg} / \mathrm{kg}$ pramipexole from saline. When the $n$ differed from the value indicated in the legend for any test condition, that $n$ is indicated by the corresponding point on the graph

the doses tested (Fig. 1, bottom panel). Linear regression analyses indicated a significant reduction in rate with pramipexole $[F(1,38)=93.3, p<0.001]$, sumanirole $[F(1$, $33)=36.3, p<0.001]$, quinpirole $[F(1,22)=26.2, p<0.001]$, and 7-OH-DPAT $[F(1,22)=42.6, p<0.001]$ (see Table 3 for ED50 values).

In the rats trained to discriminate $0.1 \mathrm{mg} / \mathrm{kg}$ pramipexole from saline, each of the antagonists tested engendered some pramipexole-appropriate responding when administered alone (Fig. 2, top panel). The $1.0 \mathrm{mg} / \mathrm{kg} \mathrm{L}-741,626$ stimulus produced over $80 \%$ pramipexole responding, while the $32 \mathrm{mg} / \mathrm{kg}$ PG01037 and $0.032 \mathrm{mg} / \mathrm{kg}$ haloperidol stimuli each produced approximately $40 \%$ pramipexole responding. When given as a pretreatment to various doses of pramipexole, $0.032 \mathrm{mg} / \mathrm{kg}$ haloperidol significantly shifted the pramipexole substitution curve to the right $[F(1,35)=$ $13.5, p<0.001]$. While $1.0 \mathrm{mg} / \mathrm{kg} \mathrm{L}-741,626$ shifted the pramipexole substitution curve down somewhat, neither this drug nor $32 \mathrm{mg} / \mathrm{kg}$ PG01037 significantly altered the pramipexole substitution curve. When given as a pretreatment, none of the antagonists significantly altered the potency at which pramipexole reduced response rates, 
Table 2 The dose of each drug in milligrams per kilogram (95\% CI in parentheses) that engendered $50 \%$ of injection-appropriate responding on the single-drug lever in each group

\begin{tabular}{lllll}
\hline & 0.1 pram vs. saline & 1.0 sum vs. saline & 0.1 pram vs. 1.0 sum or saline & 1.0 sum vs. 0.1 pram or saline \\
\hline Pramipexole & $0.050(0.011$ to 0.22$)$ & $0.13(0.041$ to 0.43$)$ & n.s. & $0.18(0.0072$ to 23$)$ \\
Sumanirole & $0.56(0.030$ to 10$)$ & $0.53(0.17$ to 1.6$)$ & n.s. & $0.68(0.20$ to 2.2$)$ \\
Quinpirole & n.s. & $0.019(0.0027$ to 0.14$)$ & Not tested & n.s. \\
7-OH-DPAT & n.s. & n.s. & Not tested & $0.022(0.0029$ to 0.22$)$ \\
Cocaine & n.s. & n.s. & Not tested & n.s. \\
\hline
\end{tabular}

ED50 values are only shown for drugs for which a linear regression indicated a significantly nonzero slope.

pram Pramipexole, sum sumanirole, n.s. not significant linear regression analysis.

although the shift observed with $1.0 \mathrm{mg} / \mathrm{kg} \mathrm{L}-741,626$ approached significance $[F(1,45)=3.54, p=0.067$; Fig. 2, bottom panel].

In the group trained to discriminate $1.0 \mathrm{mg} / \mathrm{kg}$ sumanirole from saline, all of the D2-like agonists substituted for sumanirole at some dose (Fig. 3, top panel). The slopes of the pramipexole $[F(1,34)=57.6, p<0.001]$, sumanirole $[F(1$, $37)=72.0, p<0.001]$, and quinpirole $[F(1,22)=15.1, p<$ $0.001]$ substitution curves were significantly nonzero (see Table 2 for ED50 values). The substitution profile for 7-OHDPAT was not dose dependent, and cocaine did not fully substitute for sumanirole at any dose tested. Each of the D2like agonists dose dependently reduced rates of responding, while cocaine did not alter response rates at the doses tested (Fig. 3, bottom panel). Linear regression analyses indicated a significant reduction in rate with pramipexole $[F(1,38)=$ $31.7, p<0.001]$, sumanirole $[F(1,38)=20.7, p<0.001]$, quinpirole $[F(1,22)=22.6, p<0.001]$, and 7-OH-DPAT $[F(1,22)=51.8, p<0.001$; see Table 3 for ED50 values].

In the rats trained to discriminate $1.0 \mathrm{mg} / \mathrm{kg}$ sumanirole from saline, each of the antagonists tested engendered some sumanirole-appropriate responding when administered alone (Fig. 4, top panel). The $32 \mathrm{mg} / \mathrm{kg}$ PG01037 stimulus produced over $80 \%$ sumanirole responding, while the $1.0 \mathrm{mg} / \mathrm{kg} \mathrm{L}-741,626$ and $0.032 \mathrm{mg} / \mathrm{kg}$ haloperidol stimuli each produced approximately 40\% sumanirole responding. However, this group responded approximately $30 \%$ of the time on the sumanirole nose-poke when given a saline pretreatment. When given as a pretreatment to various doses of sumanirole, none of the antagonists had a significant effect. When given as a pretreatment, $32 \mathrm{mg} / \mathrm{kg}$ PG01037 did not significantly alter the response rate suppression seen with sumanirole administration (Fig. 4, bottom panel). However, $1.0 \mathrm{mg} / \mathrm{kg} \mathrm{L}-741,626[F(1,45)=14.7, p<0.001]$ and 0.032 $\mathrm{mg} / \mathrm{kg}$ haloperidol $[F(1,45)=8.31, p=0.006]$ did shift the response rate dose-response curve to the right.

In the group trained to discriminate $1.0 \mathrm{mg} / \mathrm{kg}$ sumanirole from either $0.1 \mathrm{mg} / \mathrm{kg}$ pramipexole or saline, sumanirole, pramipexole, and 7-OH-DPAT fully substituted for sumanirole at some dose, while quinpirole produced a partial substitution curve that did not appear dose dependent (Fig. 5, top panel). The slopes of the pramipexole $[F(1,33)=8.63, p=$ $0.006]$, sumanirole $[F(1,38)=63.3, p<0.001]$, and $7-\mathrm{OH}-$ DPAT $[F(1,22)=13.5, p=0.001]$ substitution curves were significantly nonzero (see Table 2 for ED50 values). Each of the D2-like agonists dose dependently reduced rates of responding, while cocaine did not alter response rates at the doses tested (Fig. 5, bottom panel). Linear regression analyses indicated a significant reduction in rate with pramipexole $[F(1$, $38)=111, p<0.001]$, sumanirole $[F(1,38)=61.2, p<0.001]$, quinpirole $[F(1,22)=36.4, p<0.001]$, and 7-OH-DPAT $[F(1$, 22) $=19.4, p<0.001$; see Table 3 for ED50 values].

In the rats trained to discriminate $1.0 \mathrm{mg} / \mathrm{kg}$ sumanirole from either $0.1 \mathrm{mg} / \mathrm{kg}$ pramipexole or saline, two of the antagonists tested engendered some sumanirole-appropriate responding when administered alone (Fig. 6, top panel). The $32 \mathrm{mg} / \mathrm{kg}$ PG01037 stimulus produced approximately $60 \%$ sumanirole responding, while the $0.032 \mathrm{mg} / \mathrm{kg}$

Table 3 The dose of each drug in milligrams per kilogram (95\% CI in parentheses) that reduced response rates to $50 \%$ of saline response rates for each group

\begin{tabular}{lllll}
\hline & 0.1 pram vs. saline & 1.0 sum vs. saline & 0.1 pram vs. 1.0 sum or saline & 1.0 sum vs. 0.1 pram or saline \\
\hline Pramipexole & $0.11(0.040$ to 0.64$)$ & $0.18(0.051$ to 0.70$)$ & $0.28(0.094$ to 1.0$)$ & $0.19(0.080$ to 0.49$)$ \\
Sumanirole & $1.8(0.40$ to 9.1$)$ & $4.1(0.52$ to 66$)$ & $4.0(1.2$ to 19$)$ & $2.8(0.85$ to 11$)$ \\
Quinpirole & $0.024(0.0062$ to 0.10$)$ & $0.12(0.030$ to 1.17$)$ & Not tested & $0.040(0.014$ to 0.15$)$ \\
7-OH-DPAT & $0.037(0.013$ to 0.12$)$ & $0.040(0.016$ to 0.11$)$ & Not tested & $0.046(0.010$ to 0.36$)$ \\
Cocaine & n.s. & n.s. & Not tested & n.s.
\end{tabular}

Cocaine did not reduce response rates to $50 \%$ of control values in any group.

pram Pramipexole, sum sumanirole, n.s. not significant linear regression analysis. 
0.1 Pramipexole | Saline
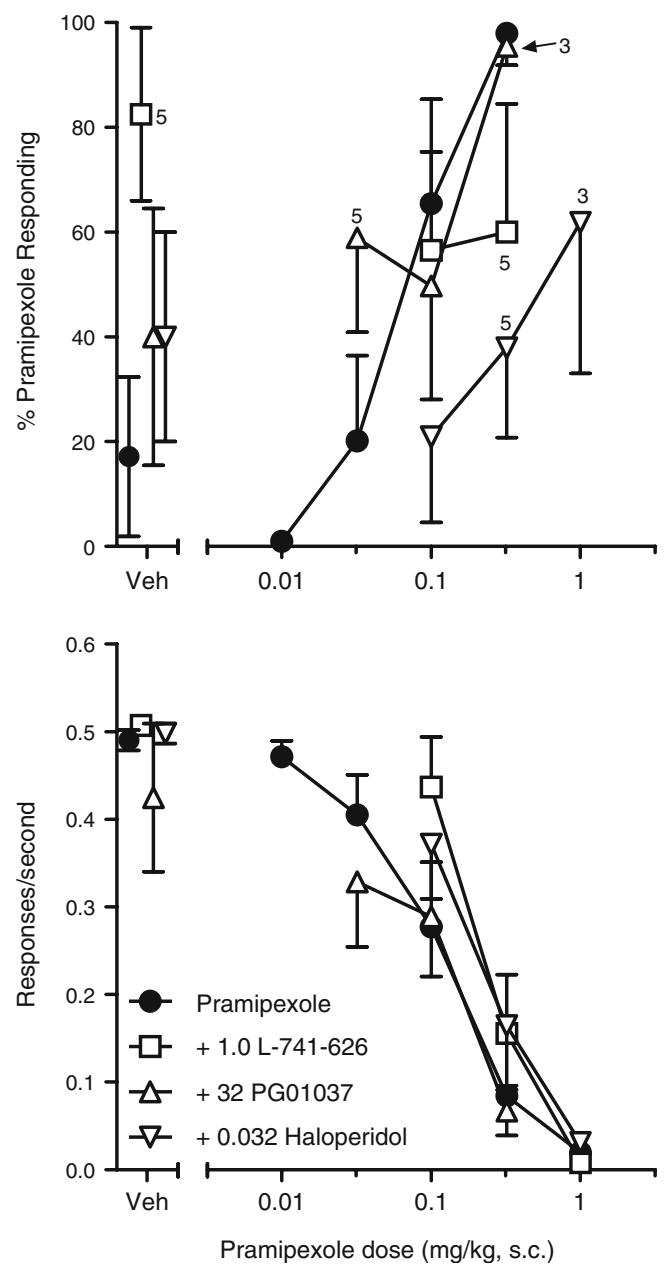

Fig. 2 Antagonism of the discriminative stimulus and rate decreasing effects of pramipexole in rats trained to discriminate $0.1 \mathrm{mg} / \mathrm{kg}$ pramipexole from saline. Open symbols represent the administration of the dose of pramipexole indicated with a 30-min pretreatment of $1.0 \mathrm{mg} / \mathrm{kg} \mathrm{L}-741,626$ (squares), $32 \mathrm{mg} / \mathrm{kg}$ PG01037 (triangles), or $0.032 \mathrm{mg} / \mathrm{kg}$ haloperidol (inverted triangles). Open symbols above Veh represent data from the antagonist given as a pretreatment to a saline injection. Unless indicated on the graph, each treatment condition represents $n=6$

haloperidol stimulus produced approximately $35 \%$ sumanirole responding. When given as a pretreatment to various doses of sumanirole, none of the antagonists had a significant effect. Likewise, none of the antagonists significantly altered the rate-suppression dose-response curve of sumanirole (Fig. 6, bottom panel).

Not all of the rats in the group trained to discriminate 0.1 $\mathrm{mg} / \mathrm{kg}$ pramipexole from either $1.0 \mathrm{mg} / \mathrm{kg}$ sumanirole or saline met training criteria (Table 1). Furthermore, the substitution curves of the two training drugs in the five rats that did meet criteria were not dose dependent (Fig. 7, top panel). No dose of either pramipexole or sumanirole produced more than a $40 \%$ pramipexole stimulus. The rate-suppression data were dose dependent, however. Both the pramipexole $[F(1,23)=48.0, p<0.001]$ and sumanirole $[F(1,23)=38.4, p<0.001]$ rate-suppression linear regression analyses were significantly nonzero.

The doses at which sumanirole substituted for the training drug in each group did not differ (see Table 2 for ED50 values). However, pramipexole generalized at lower doses in the $0.1 \mathrm{mg} / \mathrm{kg}$ pramipexole vs. saline group as compared with the $1.0 \mathrm{mg} / \mathrm{kg}$ sumanirole vs. saline group $[F(1,67)=7.83, p=0.007]$ or the $1.0 \mathrm{mg} / \mathrm{kg}$ sumanirole vs. $0.1 \mathrm{mg} / \mathrm{kg}$ pramipexole or saline group $[F(1,66)=4.57, p=$ $0.036]$. The potency of pramipexole to substitute for sumanirole did not differ in the two groups trained to discriminate $1.0 \mathrm{mg} / \mathrm{kg}$ sumanirole from saline or either $0.1 \mathrm{mg} / \mathrm{kg}$ pramipexole or saline. The doses at which sumanirole reduced rates of responding also did not differ among the training groups (see Table 3 for ED50 values). However, pramipexole was more potent at reducing response rates in the group trained to discriminate $0.1 \mathrm{mg} / \mathrm{kg}$ pramipexole from saline than the group trained to discriminate $0.1 \mathrm{mg} / \mathrm{kg}$ pramipexole from either $1.0 \mathrm{mg} / \mathrm{kg}$ sumanirole or saline $[F(1,62)=8.30, p=0.005]$ and the group trained to
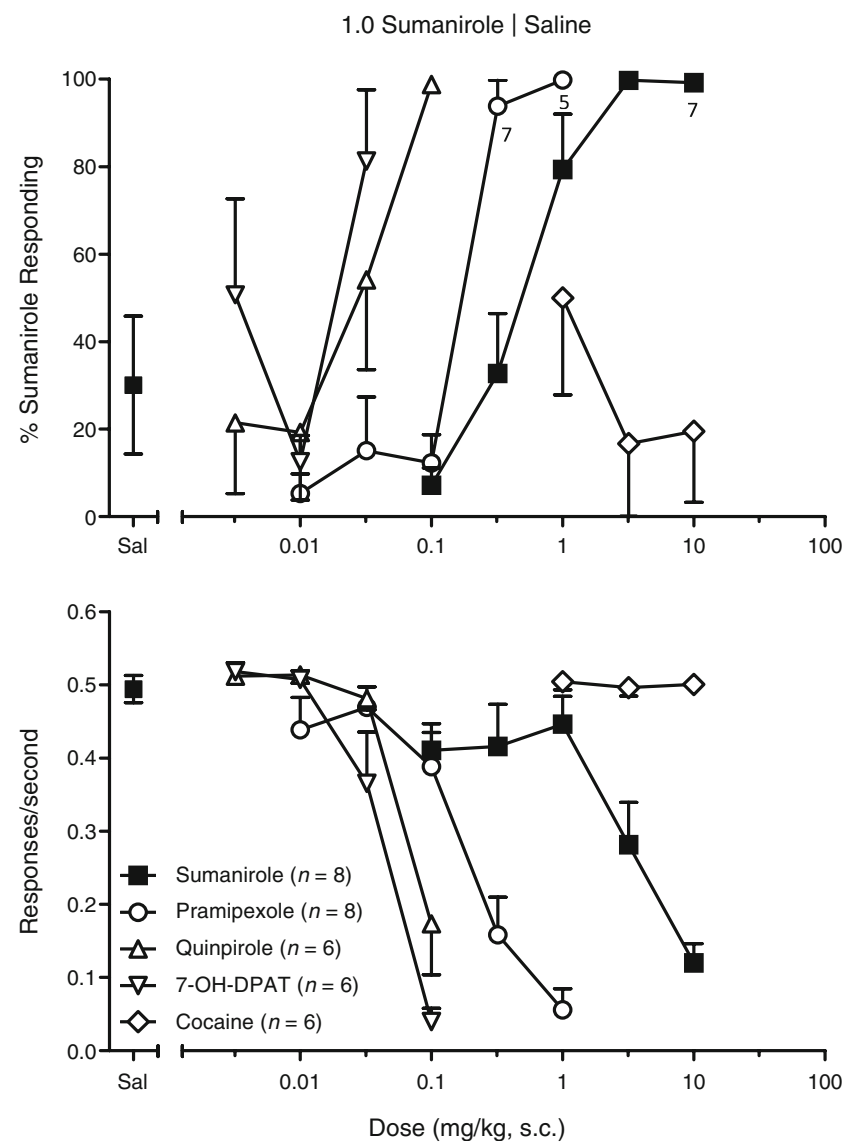

Fig. 3 Substitution and rates of responding engendered by dopamine D2-like agonists and cocaine in rats trained to discriminate $1.0 \mathrm{mg} / \mathrm{kg}$ sumanirole from saline. When the $n$ differed from the value indicated in the legend for any test condition, that $n$ is indicated by the corresponding point on the graph 

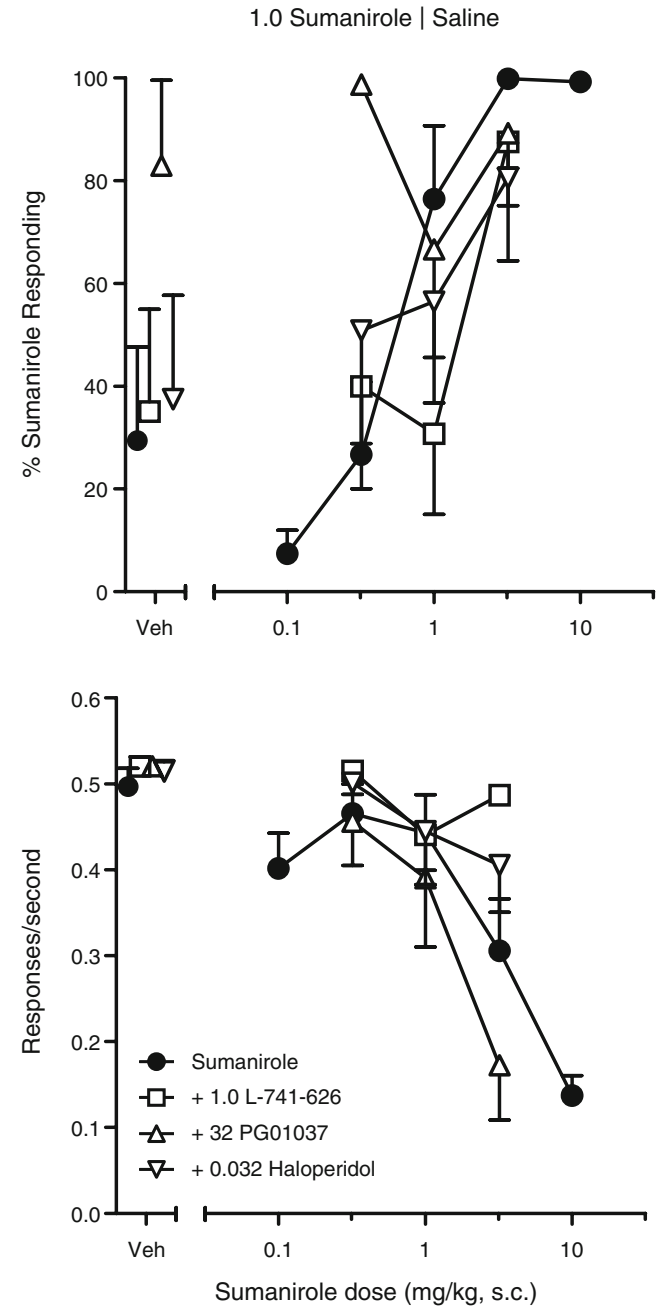

Fig. 4 Antagonism of the discriminative stimulus and rate decreasing effects of sumanirole in rats trained to discriminate $1.0 \mathrm{mg} / \mathrm{kg}$ sumanirole from saline. Open symbols represent the administration of the dose of sumanirole indicated with a 30-min pretreatment of $1.0 \mathrm{mg} / \mathrm{kg} \mathrm{L}-741,626$ (squares), $32 \mathrm{mg} / \mathrm{kg}$ PG01037 (triangles), or $0.032 \mathrm{mg} / \mathrm{kg}$ haloperidol (inverted triangles). Open symbols above Veh represent data from the antagonist given as a pretreatment to a saline injection

discriminate $1.0 \mathrm{mg} / \mathrm{kg}$ sumanirole from either $0.1 \mathrm{mg} / \mathrm{kg}$ pramipexole or saline $[F(1,77)=10.0, p=0.002]$ and nearly so in the group trained to discriminate $1.0 \mathrm{mg} / \mathrm{kg}$ sumanirole from saline $[F(1,77)=3.49, p=0.065]$.

\section{Discussion}

The current study represents the first published account of pramipexole or sumanirole being trained as discriminative stimuli. In general, each of the D2-like agonists in the current study generalized to either pramipexole or sumanirole, although some generalizations were not full or dose dependent. Other D3-preferring agonists have been trained as discriminative stimuli in the past, yielding similar results (e.g., Katz and Alling 2000; Millan et al. 2000, 2007), but sumanirole is the first highly selective D2-preferring agonist to be trained as a discriminative stimulus. It is notable that the generalization profile in the $1.0 \mathrm{mg} / \mathrm{kg}$ sumanirole vs. saline group was similar to the $0.1 \mathrm{mg} / \mathrm{kg}$ pramipexole vs. saline group in the current study.

Taken as a whole, the current results suggest that the discriminative stimulus effects of pramipexole and sumanirole are mediated by D2 receptors. The first evidence of this assertion lies with the antagonist pretreatment data. In the group trained to discriminate $0.1 \mathrm{mg} / \mathrm{kg}$ pramipexole from saline, $32 \mathrm{mg} / \mathrm{kg}$ of the D3 antagonist PG01037 did not alter the discriminative stimulus effects of pramipexole, while $1.0 \mathrm{mg} / \mathrm{kg}$ of the D2 antagonist L-741,626 reduced the pramipexole-appropriate responding following $0.32 \mathrm{mg} / \mathrm{kg}$ pramipexole from near $100 \%$ to approximately $60 \%$. The linear regression comparison did not show this effect to be significant, potentially limited by only two doses of L-741,626 being included. In the rats trained to discriminate $1.0 \mathrm{mg} / \mathrm{kg}$ sumanirole from saline, $1.0 \mathrm{mg} / \mathrm{kg} \mathrm{L}-741,626$ reduced the sumanirole substitution curve to the greatest degree, although
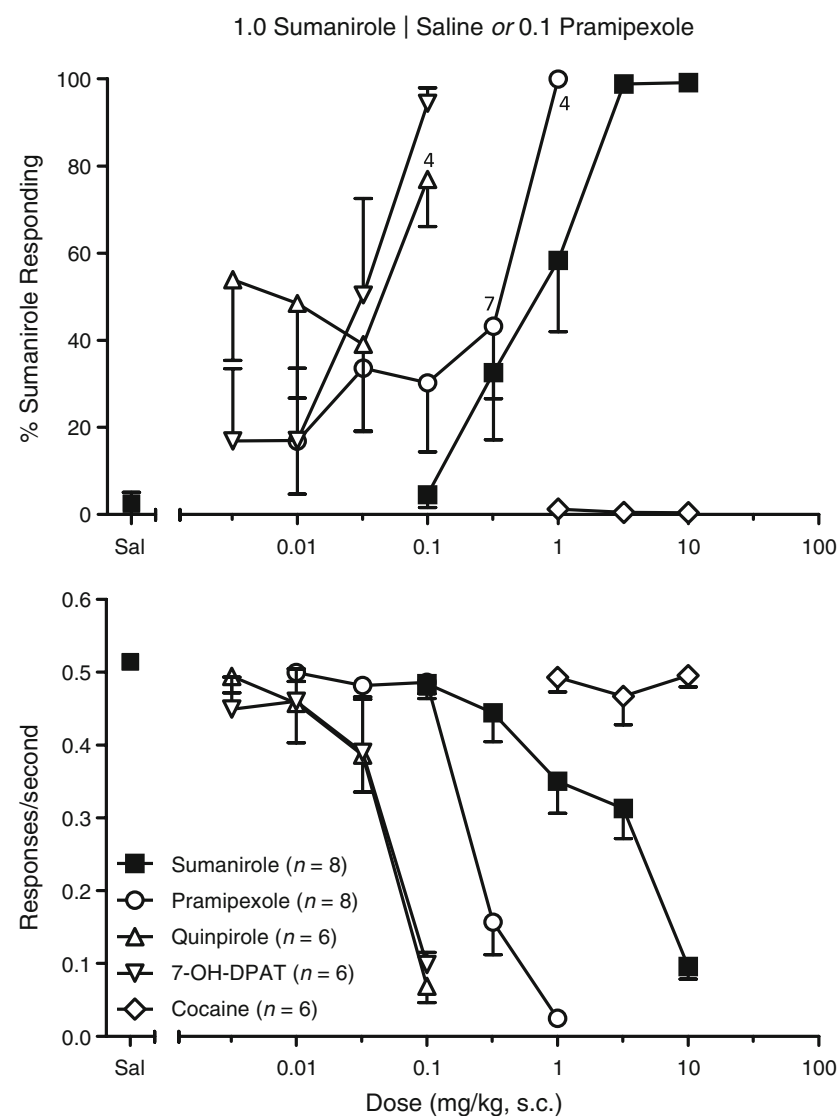

Fig. 5 Substitution and rates of responding engendered by dopamine D2-like agonists and cocaine in rats trained to discriminate $1.0 \mathrm{mg} / \mathrm{kg}$ sumanirole from either $0.1 \mathrm{mg} / \mathrm{kg}$ pramipexole or saline. When the $n$ differed from the value indicated in the legend for any test condition, that $n$ is indicated by the corresponding point on the graph 
1.0 Sumanirole | Saline or 0.1 Pramipexole
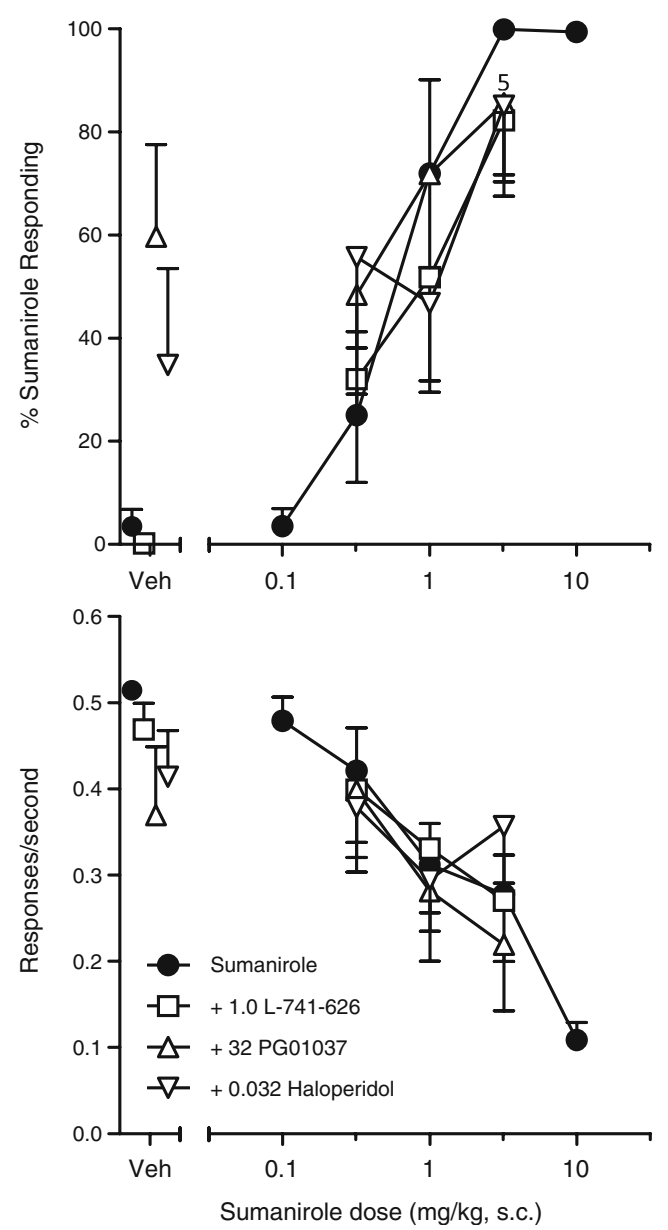

Fig. 6 Antagonism of the discriminative stimulus and rate decreasing effects of sumanirole in rats trained to discriminate $1.0 \mathrm{mg} / \mathrm{kg}$ sumanirole from either $0.1 \mathrm{mg} / \mathrm{kg}$ pramipexole or saline. Open symbols represent the administration of the dose of sumanirole indicated with a 30-min pretreatment of $1.0 \mathrm{mg} / \mathrm{kg} \mathrm{L}-741,626$ (squares), $32 \mathrm{mg} / \mathrm{kg}$ PG01037 (triangles), or $0.032 \mathrm{mg} / \mathrm{kg}$ haloperidol (inverted triangles). Open symbols above Veh represent data from the antagonist given as a pretreatment to a saline injection. Each point represents $n=6$ unless indicated

this effect did not reach significance. Both $32 \mathrm{mg} / \mathrm{kg}$ PG01037 and $1.0 \mathrm{mg} / \mathrm{kg} \mathrm{L}-741,626$ have been shown to be effective doses to antagonize other D3- and D2-mediated effects in vivo (Collins et al. 2007). This pattern of D2 antagonists having greater efficacy in reducing the discriminative stimulus effects of D2-like agonists, even those that are have preference for the D3 receptor, agrees with reports published in rats trained to discriminate S32504 (Millan et al. 2007) or 7-OH-DPAT (Christian et al. 2001) from saline. Both of these studies found that D3 antagonists either potentiated or did not alter the substitution curves of the training drug, while a D2 antagonist reduced the substitution of the training drug.
The failure to train and maintain a discrimination with the training condition that paired one response option with $0.1 \mathrm{mg} / \mathrm{kg}$ pramipexole and the other with either $1.0 \mathrm{mg} / \mathrm{kg}$ sumanirole or saline is the second piece of evidence that supports the assertion that the discriminative stimulus effects of both pramipexole and sumanirole are mediated through D2 receptors. If D2 and D3 receptor activation mediate distinct discriminative stimuli, then the either/or groups trained to discriminate pramipexole or sumanirole from either saline or the other agonist both should have learned the discrimination with relative ease. However, if D2 receptor activation primarily mediates D2-like agonist discriminative stimulus effects, the current results would be expected. A dose of $0.1 \mathrm{mg} / \mathrm{kg}$ pramipexole has a small amount of D2 activity in a hypothermia assay, while $1.0 \mathrm{mg} / \mathrm{kg}$ sumanirole produces a robust hypothermia response (Collins et al. 2007). Using this hypothermia response as a gauge of in vivo $\mathrm{D} 2$ activity, the group trained to discriminate $1.0 \mathrm{mg} / \mathrm{kg}$ sumanirole from either $0.1 \mathrm{mg} / \mathrm{kg}$ pramipexole or saline was required to discriminate a large amount of D2 activation

0.1 Pramipexole | Saline or 1.0 Sumanirole
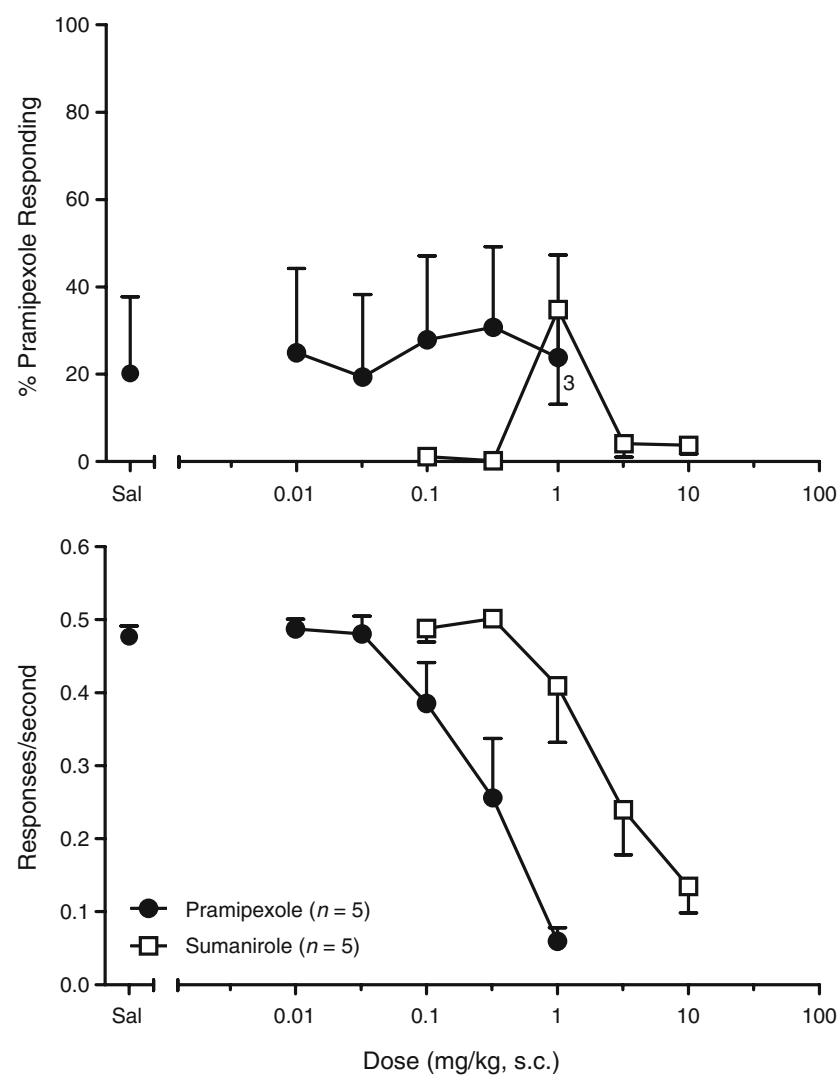

Fig. 7 Substitution and rates of responding engendered by dopamine D2-like agonists and cocaine in rats trained to discriminate $0.1 \mathrm{mg} / \mathrm{kg}$ pramipexole from either $1.0 \mathrm{mg} / \mathrm{kg}$ sumanirole or saline. Only five of eight rats in this group met training criteria, and each point is the mean of these five rats. When the $n$ differed from five for any test condition, that $n$ is indicated by the corresponding point on the graph 
( $1.0 \mathrm{mg} / \mathrm{kg}$ sumanirole) from a complex stimulus of either a small amount $(0.1 \mathrm{mg} / \mathrm{kg}$ pramipexole) or no (saline) D2 activation. High/low dose discriminations are successfully discriminated by rats, including high and low doses of cocaine (Kleven and Koek 1997, 1998) and the atypical antipsychotic clozapine (Prus et al. 2006), so it is not surprising if these rats learned to discriminate differing degrees of D2 agonist activity. However, the group trained to discriminate $0.1 \mathrm{mg} / \mathrm{kg}$ pramipexole from either $1.0 \mathrm{mg} / \mathrm{kg}$ sumanirole or saline was required to discriminate a low degree of D2 activation from a compound stimulus of a high degree of D2 activation and no D2 activation. No such discrimination within a single receptor subclass has been trained to the authors' knowledge, and it seems likely that such a complex discrimination would be difficult to learn and maintain.

These assertions regarding levels of in vivo activation of D2 and D3 receptors corresponding to specific doses of pramipexole and sumanirole rely on the unproven hypothesis that the doses of these drugs required to produce yawning and hypothermia are roughly equivalent to the doses required to produce a discriminative stimulus. It is not known which area or areas of the brain mediate the discriminative stimulus effects of D2-like agonists, but the nucleus accumbens seems to be critically involved in cocaine's discriminative stimulus effects (Callahan et al. 1997). This contrasts with the induction of yawning and hypothermia, which are probably mediated by nuclei in the hypothalamus (Argiolas and Melis 1998, Parada et al. 1995, but see Barik and de Beaurepaire 1998). D3 receptors have a more limited distribution than do D2 receptors, and they are more often located on presynaptic sites (Bouthenet et al. 1991). How such distributions may be important to the discriminative stimulus effects of compounds acting at these receptors has not been studied in any detailed way, however.

The number of sessions required to meet training criteria is the final set of data that supports the notion that D2 activation was the primary basis for the discriminations learned in each group. The group trained to discriminate $1.0 \mathrm{mg} / \mathrm{kg}$ sumanirole from saline met training criteria much more quickly than the other three groups. Given the D2-mediation hypothesis, this training condition is the most straightforward, requiring animals to discriminate the distinct stimuli of a high degree of D2 activation and no D2 activation. The $0.1 \mathrm{mg} / \mathrm{kg}$ pramipexole vs. saline and $1.0 \mathrm{mg} / \mathrm{kg}$ sumanirole vs. $0.1 \mathrm{mg} / \mathrm{kg}$ pramipexole or saline groups require discriminations between less distinct stimuli, and these groups required more training sessions to meet training criteria. The group trained to discriminate $0.1 \mathrm{mg} / \mathrm{kg}$ pramipexole from either $1.0 \mathrm{mg} / \mathrm{kg}$ sumanirole or saline required slightly more training sessions to meet criteria in those rats that eventually met criteria. The five rats that met training criteria also met test criteria considerably less often, only finishing sumanirole and pramipexole dose-response curves before the termination of the experiment. Furthermore, the substitution data in this group was not dose dependent, indicating that the response patterns of these rats were not based on the successful discrimination between $0.1 \mathrm{mg} / \mathrm{kg}$ pramipexole and either $1.0 \mathrm{mg} / \mathrm{kg}$ sumanirole or saline. Instead, throughout the hundreds of sessions in the current experiment, it is likely that some rats occasionally met test criteria in this group largely due to chance.

While the current study and other research (Bristow et al. 1998; Christian et al. 2001; Millan et al. 2000, 2007) suggest that the discriminative stimulus effects of D2-like agonists are mediated primarily through D2 receptors, it remains an open question whether D3 receptor activation produces any discriminative stimulus effects under any condition. Should a highly selective D3 agonist with no appreciable D2 affinity become available (e.g., Wang et al. 2008), this question could be addressed more conclusively. In addition, the substitution profiles between the group trained to discriminate $1.0 \mathrm{mg} / \mathrm{kg}$ sumanirole from saline and the group trained to discriminate $0.1 \mathrm{mg} / \mathrm{kg}$ pramipexole from saline are not identical, suggesting D3 receptor activation may be play a role. The most striking difference is in the substitution data obtained with the dopamine antagonists administered prior to a saline injection. In each of these groups, the antagonist selective for the opposite receptor as the training agonist substituted at over $80 \%$. While also coinciding with research suggesting D2 and D3 receptors have opposing actions in certain systems (e.g., Collins et al. 2007), these data point to qualitatively different stimuli being discriminated by these two groups. It is unknown whether this pattern is related to D3 activation or differing levels D2 activation. This issue could be addressed by training two groups of rats; one to discriminate $1.0 \mathrm{mg} / \mathrm{kg}$ sumanirole from saline and the other to discriminate a dose of sumanirole that has approximately equivalent D2 activity as $0.1 \mathrm{mg} / \mathrm{kg}$ pramipexole (using the hypothermia data of Collins et al. 2007, this would be approximately $0.18 \mathrm{mg} / \mathrm{kg}$ sumanirole). Such a pair of training groups could be employed to address whether the pattern of D2 and D3 antagonist substitution data is related to degree of D2 activation or is the result of a D3-mediated discriminative stimulus.

Cocaine did not substitute for either pramipexole or sumanirole in any of the groups in which it was tested. In the group trained to discriminate $1.0 \mathrm{mg} / \mathrm{kg}$ sumanirole from a rotation of $0.1 \mathrm{mg} / \mathrm{kg}$ pramipexole and saline, all the rats responded on the pramipexole/saline lever after cocaine administration. From these data, it cannot be determined whether the cocaine stimulus approximated saline or pramipexole. However, cocaine did not substitute for pramipexole in the rats trained to discriminate $0.1 \mathrm{mg} / \mathrm{kg}$ pramipexole from saline, so it could be assumed that cocaine did not 
approximate the pramipexole stimulus in either group. This coincides with past research showing no substitution of cocaine in rats trained to discriminate the D2-like agonists 7-OH-DPAT (Varty and Higgins 1997), PD 128,907 (Bristow et al. 1998), quinpirole (Katz and Alling 2000), and apomorphine (Tang and Franklin 1987). However, at least one study found partial substitution of cocaine to a 7-OHDPAT or PD 128,907 stimulus (Katz and Alling 2000).

The rate suppressant effects of sumanirole did not differ among the training groups, but pramipexole was most potent with this endpoint in the animals trained to discriminate $0.1 \mathrm{mg} / \mathrm{kg}$ pramipexole from saline. Like with the stimulus effects, the rate suppressant effects of these drugs also appear to be mediated through D2 receptors. L741,626 was most effective at antagonizing the rate suppressant effects of pramipexole in the rats trained to discriminate $0.1 \mathrm{mg} / \mathrm{kg}$ pramipexole from saline and sumanirole in the rats trained to discriminate $1.0 \mathrm{mg} / \mathrm{kg}$ sumanirole from saline. PG01037 had no effect on the rate suppressant effects of either of these compounds.

In conclusion, the discriminative stimulus and rate suppressant effects of the D2-like agonists pramipexole and sumanirole appear to be mediated through D2 rather than D3 receptors. This is despite the very different selectivity profiles of these two drugs for D2 or D3 receptors. It may also be the case that $\mathrm{D} 3$ receptor activation is not associated with a distinct discriminative stimulus, although this cannot be determined conclusively without more selective compounds.

Acknowledgements The authors would like to thank Gail Winger and Emily Jutkiewicz for advice and guidance throughout the experiment and with manuscript preparation. The authors also thank Alexa Cohen, Simon Cohen, Antwan Hall, Eugene Kligman, Elizabeth Kossak, Jennifer Montgomery, Nathaniel Reichwage, and Emily West for technical assistance. This research was supported by USPHS/NIDA grants R01 DA020669 and T32 DA007267. The content is solely the responsibility of the authors and does not necessarily represent the official views of the National Institute on Drug Abuse or the National Institutes of Health.

\section{References}

Argiolas A, Melis MR (1998) The neuropharmacology of yawning. Eur J Pharmacol 343:1-16

Barik S, de Beaurepaire R (1998) Hypothermic effects of dopamine $\mathrm{D}_{3}$ receptor agonists in the island of Calleja magna. Potentiation by $\mathrm{D}_{1}$ activation. Pharmacol Biochem Behav 60:313-319

Bouthenet ML, Souil E, Martres MP, Sokoloff P, Giros B, Schwartz JC (1991) Localization of $\mathrm{D}_{3}$ receptor mRNA in the rat brain using in situ hybridization histochemistry: comparison with dopamine $\mathrm{D}_{2}$ receptor mRNA. Brain Res 564:203-219

Bristow LJ, Cook GP, Patel S, Curtis N, Mawer I, Kulagowski JJ (1998) Discriminative stimulus properties of the putative dopamine $\mathrm{D}_{3}$ receptor agonist, (+)-PD 128907: role of presynaptic dopamine $\mathrm{D}_{2}$ autoreceptors. Neuropharmacology 37:793-802
Callahan PM, Garza DLG II, Cunningham KA (1997) Mediation of the discriminative stimulus properties of cocaine by mesocorticolimbic dopamine systems. Pharmacol Biochem Behav 57:601-607

Caul WF, Barrett RJ, Huffman EM, Stadler JR (1996) Rebound responding following a single dose of drug using an amphetaminevehicle-haloperidol drug discrimination. Psychopharmacology (Berl) 128:274-279

Christian AJ, Goodwin AK, Baker LE (2001) Antagonism of the discriminative stimulus effects of (+)-7-OH-DPAT by remoxipride but not PNU-99194A. Pharmacol Biochem Behav 68:371-377

Collins GT, Newman AH, Grundt P, Rice KC, Husbands SM, Chauvignac C, Chen J, Wang S, Woods JH (2007) Yawning and hypothermia in rats: effects of dopamine D3 and D2 agonists and antagonists. Psychopharmacology (Berl) 193:159-170

Grundt P, Husbands SLJ, Luedtke RR, Taylor M, Newman AH (2007a) Analogues of the dopamine D2 receptor antagonist L741,626: binding, function, and SAR. Bioorg Med Chem Lett 17:745-749

Grundt P, Prevatt KM, Cao J, Taylor M, Foresca CZ, Choi J, Jenkins BG, Luedke RR, Newman AH (2007b) Heterocyclic analogues of N-(4-(4-(2,3-dichlorophenyl)piperazin-1-yl)butyl)arylcarboxamides with functionalized linking chains as novel dopamine D3 receptor ligands: potential substance abuse therapeutic agents. J Med Chem 50:4135-4146

Katz JL, Alling KL (2000) Discriminative stimulus effects of putative D3 dopamine receptor agonists in rats. Psychopharmacology (Berl) 11:483-493

Kleven MS, Koek W (1997) Discriminative stimulus properties of cocaine: enhancement by $\beta$-adrenergic receptor antagonists. Psychopharmacology (Berl) 131:307-312

Kleven MS, Koek W (1998) Discriminative stimulus properties of cocaine: enhancement by monoamine reuptake blockers. J Pharmacol Exp Ther 284:1015-1025

Koek W, Carter LP, Lamb RJ, Chen W, Wu H, Coop A, France CP (2005) Discriminative stimulus effects of $\gamma$-hydroxybutyrate (GHB) in rats discriminating GHB from baclofen and diazepam. J Pharmacol Exp Ther 314:170-179

McCall RB, Lookingland KJ, Bédard PJ, Huff RM (2005) Sumanirole, a highly dopamine $\mathrm{D}_{2}$-selective receptor agonist: in vitro and in vivo pharmacological characterization and efficacy in animal models of Parkinson's disease. J Pharmacol Exp Ther 314:1248-1256

Millan MJ, Girardon S, Monneyron S, Dekeyne A (2000) Discriminative stimulus properties of the dopamine $\mathrm{D}_{3}$ receptor agonists, PD128,907 and 7-OH-DPAT: a comparative characterization with novel ligands at $D_{3}$ versus $D_{2}$ receptors. Neuropharmacology 39:586-598

Millan MJ, Iob L, Péglion J, Dekeyne A (2007) Discriminative stimulus properties of $\mathrm{S} 32504$, a novel $\mathrm{D}_{3} / \mathrm{D}_{2}$ receptor agonist and antiparkinsonian agent, in rats: attenuation by the antipsychotics, aripiprazole, bifeprunox, $N$-desmethylclozapine, and by selective antagonists at dopamine $\mathrm{D}_{2}$ but not $\mathrm{D}_{3}$ receptors. Psychopharmacology (Berl) 191:767-782

Motulsky HJ, Christopoulos A (2003) Fitting models to biological data using linear and nonlinear regression: a practical guide to curve fitting. GraphPad Software, San Diego

Newman-Tancredi A, Cussac D, Audinot V, Nicolas J, de Ceuninck F, Boutin J, Millan MJ (2002) Differential actions of antiparkinson agents at multiple classes of monaminergic receptor. II. Agonist and antagonist properties at subtypes of dopamine $\mathrm{D}_{2}$-like receptor and $\alpha_{1} / \alpha_{2}$-adrenoceptor. J Pharmacol Exp Ther 303:805-814

Overton DA (1982) Multiple drug training as a method for increasing the specificity of the drug discrimination procedure. J Pharmacol Exp Ther 221:166-172

Parada MA, Puig de Parada M, Rada P, Hernandez L (1995) Sulpiride increases and dopamine decreases intracranial temperature in rats 
when injected in the lateral hypothalamus: an animal model for the neuroleptic malignant syndrome? Brain Res 674:117-121

Prus AJ, Philibin SD, Pehrson AL, Porter JH (2006) Discriminative stimulus properties of the atypical antipsychotic drug clozapine in rats trained to discriminate $1.25 \mathrm{mg} / \mathrm{kg}$ clozapine vs. $5.0 \mathrm{mg} / \mathrm{kg}$ clozapine vs. vehicle. Behav Pharmacol 17:185-194

Tang AH, Franklin SR (1987) Discriminative stimulus effects of a low dose of apomorphine in the rat. Psychopharmacology (Berl) 91:61-66
Varty GB, Higgins GA (1997) Investigations into the nature of a 7-OH-DPAT discriminative cue: comparisons with $d$-amphetamine. Eur J Pharmacol 339:101-107

Wang S, Chen J, Gollins GT, Zhang J, Yang C, Levant B, Woods JH (2008) Design, synthesis, and evaluation of highly selective dopamine D3 ligands with a novel profile of pharmacological and behavioral selectivity. J Med Chem (in press)

Zar JH (1999) Biostatistical analysis, 4th edn. Prentice Hall, Upper Saddle River 